\title{
Effect of Mechanical Stresses on Graphene-Based Devices
}

\author{
Yi Zheng, Guido Valerio, and Zhuoxiang Ren \\ Sorbonne Universités, UPMC Univ Paris 06 UR2, L2E, F-75005 Paris, France
}

\begin{abstract}
SUMMARY
In this work, we analyse the effect of mechanical stresses on graphene devices for application in flexible electronics. The possible Schottky contacts in the transistor are modeled through suitable transmission coefficients describing the tunnelling through the electrostatic potential along the graphene channel. The surface potential is determined by imposing an equality between the charges computed with a micro- and a macro-model. All these computation tools are explicitly dependent on the choice of geometrical parameters, thus allowing the description of uniform geometrical deformations due to strains along a given direction. Numerical results are computed and discussed. Copyright (c) 2016 John Wiley \& Sons, Ltd.
\end{abstract}

Received ..

KEY WORDS: Graphene; Geometrical deformations; Energy bands; Numerical methods; Electrostatics

\section{INTRODUCTION}

The design of novel electronic devices for diverse applications, such as biomedical, security or leisure, must face several challenges, notably in terms of flexibility, biocompatibility, and low power consumption. In this framework, graphene-based transistors are currently regarded as an attractive solution of these issues.

Graphene is a two dimensional material consisting of hexagonal carbon atoms arranged in honeycomb lattice [1]-[2]. Its distinctive and special electronic properties, namely its high electronic mobility, have attracted much research interest in the last years. Furthermore, its mechanical properties, namely its elasticity and robustness, make it an ideal candidate for integration in flexible circuits.

Unfortunately, graphene sheet exhibit no band gap, and for this reason cannot be directly used in electronic devices such as field-effect transistors (FET). Different methods to tune the band gap of graphene have been proposed for practical application. The most appealing approach, compatible with the realization of fully planar nanotransistors, is the cutting of graphene sheets into long and thin nanoribbons, exhibiting a band gap directly related to their width, and then to the number of atoms along their shorter dimension. Recently, FET devices using a graphene nanoribbon of dimensions $7 \times 3 \mu \mathrm{m}^{2}$ on a $\mathrm{SiO}_{2}$ were reported in [3]. Electric contacts were fabricated by means of a transmission electron microscopy grid as a shadow mask, to avoid the exposure of the graphene to chemical solvents.

Of course, a tool for the reliable modelling of graphene-based devices, relating macroscopic voltages and currents, is necessary for their design. Specifically, such a simulation tool would allow the evaluation of the effects of variations due to fabrication inaccuracies or physical external constraints.

*Correspondence to: yi.zheng@upmc.fr, guido.valerio@upmc.fr, zhuoxiang.ren@upmc.fr 


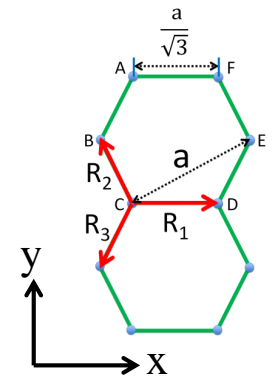

(a)

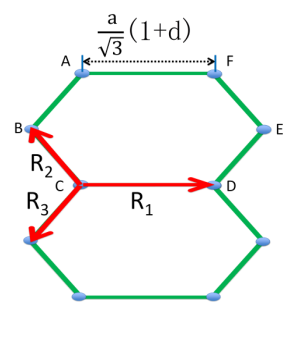

(b)

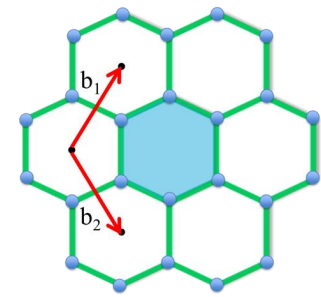

(c)

Figure 1. (a) Schematic illustration of graphene cell. (b) Effect of strain on graphene cells. (c) Reciprocal lattice of graphene: $b_{1}$ and $b_{2}$ are reciprocal lattice vectors and the first Brillouin zone is shaded.

While works have been performed on first-principle and experimental study of deformations on graphene sheets [4]-[6], the same efforts have not been devoted so far to the study of deformed configurations of graphene nanoribbons. In [7], a spatially periodically distributed strains is shown through first-principle calculations to be able to modify the energy gap of nanoribbons in order to overcome technological limitations currently encountered in the fabrication of large-gap devices. Other works have been focused on the effect of strains on nanoribbons through first-principle formulation [8]-[11], a very powerful method indeed, but not quite flexible and efficient to perform parametric studies of an entire transistor or to optimize a group of them.

To this aim, an efficient semi-analytical model has been proposed in [12] to model graphenebased FETs, including Schottky-barrier (SB) contacts and far-from-equilibrium carrier transport. An electrostatic analysis must be coupled to a quantum-mechanics description, yielding the charge distribution along the channel. However, the effect of non-idealities has not yet been addressed with this semi-analytic formalism. For instance, if the device is realized on flexible or stretchable substrates, mechanical stresses will inevitably provoke non-negligible geometrical deformations on the device.

In this paper, the outlined semi-analytic method is applied to characterize electronic characteristics of graphene-based FET with longitudinal (i.e., along the source-drain direction) deformations. The channel of these devices is an armchair graphene nanoribbon (aGNR). Specifically, in Section 2, the energy wavevector dispersion curve of aGNR with different deformation is described. Analytical characteristics such as effective mass and density of states for the strained aGNR are presented as well. In Section 3, the modified description of the FET is described, to be able to handle the strained device. In Section 4, performances of single ballistic transistors with both ohmic and Schottky-barrier (SB) contacts are compared under different strains.

\section{DISPERSION RELATIONS UNDER DEFORMATION}

In this section we investigate energy bands properties [13]-[16] of strained graphene and modify a simple tight-binding model for graphene nanoribbons to include the effect of strains along their longitudinal direction. We define accordingly modified effective masses and density of states to be used in the description of graphene FET in the next section.

\subsection{Energy Bands of a Graphene Sheet}

Graphene has honeycomb lattice structure and primitive cell of graphene is shown in Fig. 1. Recently there are many cases using graphene nanoribbon in field-effect transistors (FETs) (see Fig. 2). However, the electronic performance of FETs could change if a deformation of the graphene channel is present. 


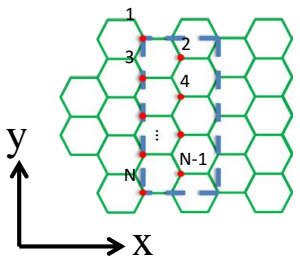

(a)

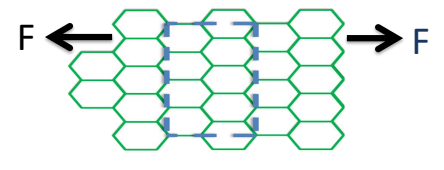

(b)

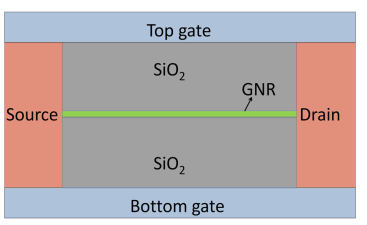

(c)

Figure 2. (a) Armchair graphene nanoribbon, (b) deformed graphene nanoribbon, (c) sectional view of a double-gate aGNR FET with deformed aGNR.

In the absence of any deformation, graphene energy bands can be computed with a tight-binding model. In the nearest-neighbor tight-binding (NNTB) model, the wave function of each electron in any unit cell only overlaps with the wave functions of its nearest neighbors [2]. Therefore, under this assumption, graphene energy bands can be computed with NNTB by using a single hopping integral, describing this first neighbor interaction. Of course, its value generally depends on the distance $\left|\mathbf{R}_{1}\right|$ between atoms, and is experimentally evaluated for non-deformed graphene sheets. The resulting energy is

$$
E^{2}(\mathbf{k})=V^{2}\left(e^{-j \mathbf{k} \cdot \mathbf{R}_{1}}+e^{-j \mathbf{k} \cdot \mathbf{R}_{2}}+e^{-j \mathbf{k} \cdot \mathbf{R}_{3}}\right)\left(e^{j \mathbf{k} \cdot \mathbf{R}_{1}}+e^{j \mathbf{k} \cdot \mathbf{R}_{2}}+e^{j \mathbf{k} \cdot \mathbf{R}_{3}}\right)
$$

where $\mathbf{k}=k_{x} \hat{\mathbf{x}}+k_{y} \hat{\mathbf{y}}$ is the reciprocal vector, and the tight-binding hopping energy is $V=2.7 \mathrm{eV}$. The hexagonal symmetry of the lattice makes it simple to express the vectors $\mathbf{R}_{1}, \mathbf{R}_{2}$, and $\mathbf{R}_{3}$ as a function of the distance $a=2.46 \AA$ (see Fig. 1). A simple replacement in (1) lead to the final expression [2]:

$$
E(\mathbf{k})= \pm V \sqrt{1+4 \cos \left(\frac{\sqrt{3} a}{2} k_{x}\right) \cos \left(\frac{a}{2} k_{y}\right)+4 \cos ^{2}\left(\frac{a}{2} k_{y}\right)}
$$

The so-obtained energy bands demonstrate the absence of energy gaps at each $\mathrm{K}$ point of the Brillouin zones, corresponding to a degeneracy of Fermi surfaces to single points. This is one of the key features to understand the physical properties of graphene [17], [2]. At the same time, the lack of an energy-gap demands the modification of graphene sheets for applications where semiconducting properties are required (i.e., nanotransistors).

\subsection{Energy Bands of a Deformed Graphene Nanoribbon}

An aGNR is obtained by cutting a graphene sheet along a given direction (see Fig. 2). Since the resulting strip lacks the translational symmetry along one direction, no simple closed form can be obtained for its energy bands as in the infinite-graphene case. However, if an ideal Dirichlet condition is enforced on the wavefunction $\Psi$ at the opposite boundaries of the ribbon of width $w=(N-1) a / 2$, where $N$ is the number of tightly bound atoms in the direction of the ribbon width ( $y$ in Fig. 2),

$$
\Psi\left(y=-\frac{a}{2}\right)=\Psi\left(y=w+\frac{a}{2}\right)=0
$$

simple conditions can be derived on the $k_{y}$ wavenumber for aGNR:

$$
(w+a) k_{y, \alpha}=\alpha \pi \quad \Rightarrow \quad k_{y, \alpha}=\frac{\alpha \pi}{w+a}=\frac{\alpha \pi}{\frac{N-1}{2} a+a}=\frac{2 \alpha \pi}{(N+1) a}
$$

where $\alpha=1, \ldots, N$.

Once the discretized values of $k_{y, \alpha}$ are replaced in the energy (2), the sub-band structure of the nanoribbon is obtained: 


$$
E_{\alpha}\left(k_{x}\right)= \pm V \sqrt{1+4 \cos \left(\frac{\sqrt{3} a}{2} k_{x}\right) A_{\alpha}+4 A_{\alpha}^{2}}
$$

where $A_{\alpha}=\cos [\pi \alpha /(N+1)]$. Eq (5) gives the energy bands of an aGNR in the absence of any geometrical deformation.

In the presence of a relative deformation $d$, The values of $\mathbf{R}_{1}, \mathbf{R}_{2}$, and $\mathbf{R}_{3}$ will change accordingly into $\mathbf{R}_{1}^{\prime}, \mathbf{R}_{2}^{\prime}$, and $\mathbf{R}_{3}^{\prime}$ (as Fig. 1 shows). Their value can be easily computed, thus leading to a modified equation for $E_{\alpha}$, the energy under deformation. The $k_{x}$ dependence is then replaced in (5) into $(1+d) k_{x}$. On the one hand, regarding the $k_{y}$ dependence, the width $w$ in (3)-(4) is deformed into $w^{\prime}=w(1-\nu d)$, and the discretized values for $k_{y}$ are scaled accordingly as $k_{y, \alpha}^{\prime}=k_{y, \alpha} /(1-\nu d)$. The argument of the cosinus in the $A_{\alpha}$ term is then unchanged due to the multiplication between $k_{y, \alpha}^{\prime}$ and the modified transverse dimension $a(1-\nu d) / 2$. The final energy bands of the nanoribbon deformed along the $x$ dimension is then

$$
E_{\alpha}^{\prime}\left(k_{x}\right)= \pm V \sqrt{1+4 \cos \left[(1+d) \frac{\sqrt{3} a}{2} k_{x}\right] A_{\alpha}+4 A_{\alpha}^{2}} .
$$

An important limitation of (6) should be stressed: no energy-gap variation could be detected with this approach, as can be done with first-principle formulations. This depends on the fact that the hopping integrals describing the interaction between close atoms has been kept constant even in the presence of a deformation. In order to obtain more accurate results, the variation of hopping integrals under deformation should be taken into account, due to the presence of different distances among nearest-neighbor atoms [18]. This effect, creating a variation of energy gap with deformation, will be described in future works. For this reason, care should be recommended to use this formulas for deformation values where the energy gap can significantly decrease, thus compromising the functioning of the FET. On the other hand, the method presented here can be adapted to include results of ab-initio simulations in the equations described in next section. This will be the object of future investigations.

In the following, the band model (6) will be used together with a first-order correction $\delta E_{\alpha}^{\prime}$ based on a perturbative approach [19] taking into account a different interaction among the atoms at the edges, being at a different chemical potential with respect to the central ones, by slightly varying their mutual hopping integrals:

$$
\delta E_{\alpha}^{\prime}\left(k_{x}\right)= \pm \frac{0.12 V}{N+1} \sin ^{2}\left(\frac{\alpha \pi}{N+1}\right) \cos \left[\frac{a(1+d) k_{x}}{\sqrt{3}}\right] .
$$

where the minus sign is chosen if $N=3 p$ for an integer $p$.

The discussion presented in this section is summarized in the results of the energy bands (6), with and without edge correction (7), shown in Fig. 3 for different values of the relative deformation $d$. As can be observed, for small deformation values the deviation from the non-deformed bands is not significant. Of course, large values of deformations (which can be purposely realized) can lead to larger energy variations.

In Fig. 4, the lowest sub-bands with different aGNR deformation are selected and compared among them. This choice is motivated by the fact that the lowest bands are the most important for the conduction phenomena in FET studied in the next section.

\subsection{Approximated Expressions: Effective Mass and Density of States}

The energy-dispersion relation in the presence of deformation can be simplified by approximating the factor $\cos \left[(1+d) \frac{\sqrt{3} a}{2} k_{x}\right]$ in it through the well-known formula:

$$
\cos \left[(1+d) \frac{\sqrt{3} a}{2} k_{x}\right] \approx 1-(1+d)^{2} \frac{3 a^{2}}{8} k_{x}^{2}
$$




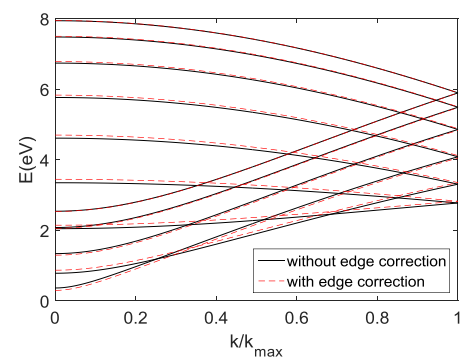

(a)

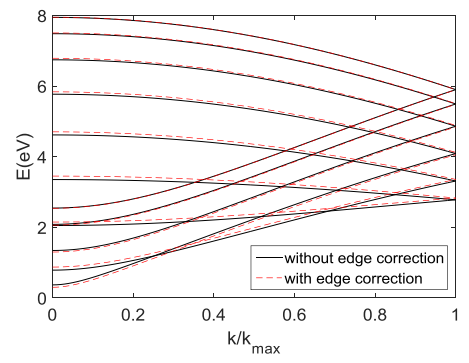

(b)

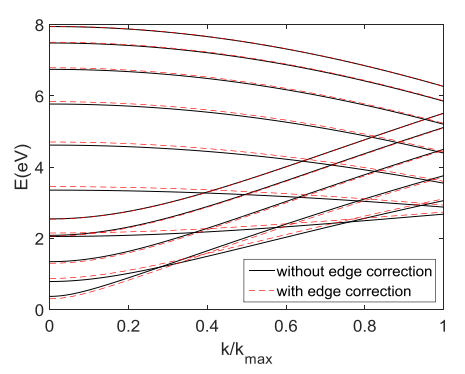

(c)

Figure 3. Comparison of the subbands of an aGNR with 12 lines between tight-binding calculation with and without edge corrections. (a) No deformation, (b) relative deformation $d=0.1$, (c) relative deformation $d=-0.1$.

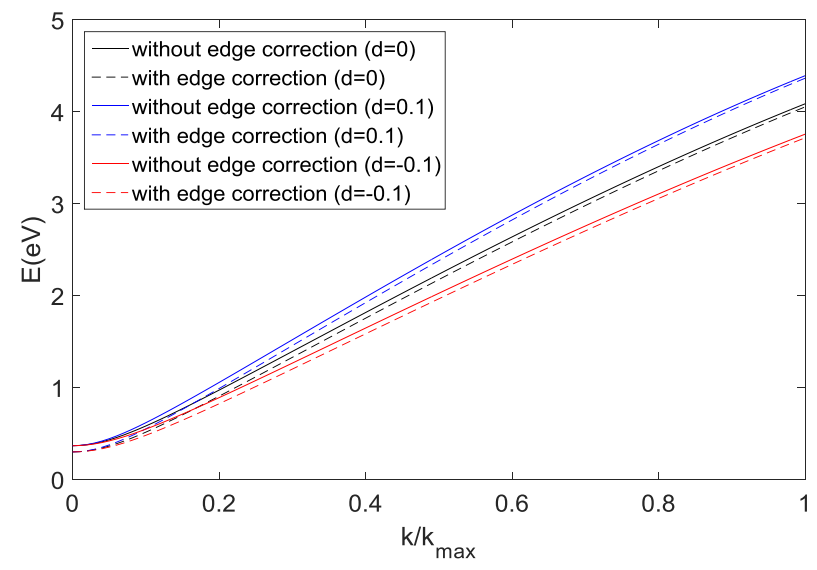

Figure 4. Comparison of the lowest subbands of an aGNR with 12 dimer lines with different deformations as explained in the legend.

Replacing (8) in (6), we obtain a simplified expression for the energy:

$$
E_{\alpha}^{\prime}\left(k_{x}\right) \approx E_{\alpha}^{\prime(2)}\left(k_{x}\right)=\sqrt{E_{\alpha}^{\prime 2}(0)+\frac{\hbar^{2} E_{\alpha}^{\prime}(0) k_{x}^{2}}{M_{\alpha}^{\prime}}}
$$

where $E_{\alpha}^{\prime}(0)$ is the energy for $k_{x}=0$, and we have identified an effective mass (EM) $M_{\alpha}^{\prime}$ in the presence of a deformation as

$$
M_{\alpha}^{\prime}=-\frac{2}{3} \frac{\hbar^{2} E_{\alpha}^{\prime}(0)}{a^{2} V^{2}(1+d)^{2} A_{\alpha}} .
$$

If a further approximation is performed on the square root in (9) $\sqrt{1+x} \approx 1+x / 2$, a simple parabolic expression for the energy is found, corresponding to a semi-classical model for the motion of carriers:

$$
E_{\alpha}^{\prime}\left(k_{x}\right) \approx E_{\alpha}^{\prime(1)}\left(k_{x}\right)=E_{\alpha}^{\prime}(0)+\frac{\hbar^{2} k_{x}^{2}}{2 M_{\alpha}^{\prime}} .
$$

The importance of the definition (10) is related to the possibility to achieve a closed-form calculation for the transmittivity $T(E)$ of a charge through a Schottky contact at the source-graphene and draingraphene interfaces. The final formulas are not reported here for brevity, but they are used in the following for the relevant computation of charges and currents [12]. 


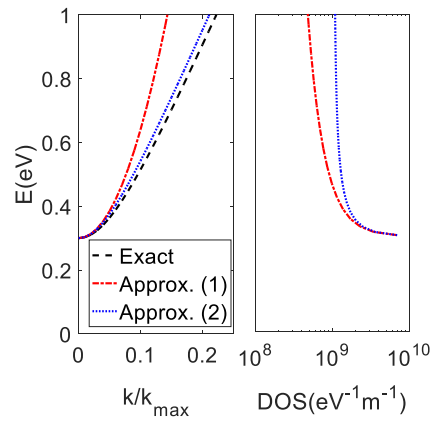

(a)

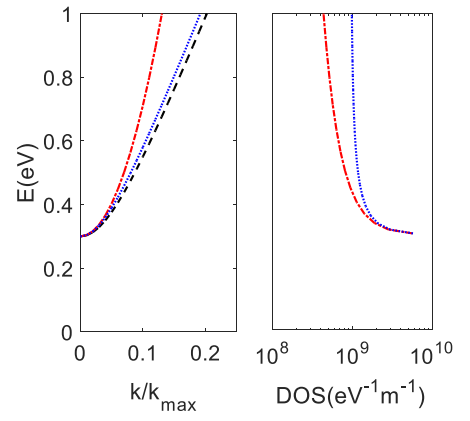

(b)

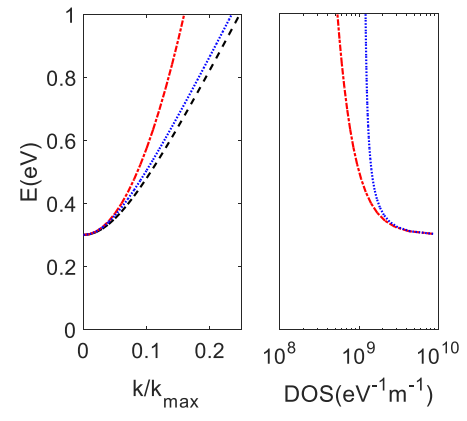

(c)

Figure 5. Energy dispersion curves and corresponding density of states of the lowest conduction subband of an aGNR with $N=12$. The analytical tight-binding result from (6) is compared with the approximate dispersions $E_{\alpha}^{\prime(1)}$ from (11) and $E_{\alpha}^{\prime(2)}$ from (9). (a) No deformation, (b) $d=0.1$, (c) $d=-0.1$.

The definition of $M_{\alpha}^{\prime}$ also allows different definitions of the density of states $D_{\alpha}^{\prime}$ [2] in the presence of deformation, according to the approximation chosen for the energy. Starting from the first-order approximation (11) we obtain

$$
D_{\alpha}^{\prime(1)}=\frac{2}{\pi \hbar} \sqrt{\frac{M_{\alpha}^{\prime}}{2\left[E_{\alpha}^{\prime(1)}\left(k_{x}\right)-E_{\alpha}^{\prime}(0)\right]}}
$$

Starting from the second-order approximation (9), we obtain

$$
D_{\alpha}^{\prime(2)}=\frac{2}{\pi \hbar} \sqrt{\frac{M_{\alpha}^{\prime}}{E_{\alpha}^{\prime}(0)}} \frac{E_{\alpha}^{\prime(2)}\left(k_{x}\right)}{\sqrt{\left[E_{\alpha}^{\prime(2)}\left(k_{x}\right)\right]^{2}-E_{\alpha}^{\prime 2}(0)}}
$$

The definition of the density of states is useful in order to compute electron density carrier by using Fermi-Dirac distributions functions. This will be accomplished in next section, where current and charge quantities will be discussed.

The relations discussed in this subsection are summarized in Fig. 5. In Fig. 5(a), the tight-binding energy dispersion together with its approximations $E^{\prime(1)}$ and $E^{\prime(2)}$ and the corresponding density of states are shown in the absence of any deformation. The same results are shown in Figs. 5(b) and (c) for a deformation $d=0.1$ and $d=-0.1$, respectively. All the numerical results are computed for an aGNR with $N=12$.

\section{SCHOTTKY FIELD-EFFECT TRANSISTORS}

Once the electronic properties of graphene nanoribbons under a deformation are determined, the behavior of the FET shown in Fig. 2 can be derived. The drain-source current $I_{\mathrm{ds}}=I_{\mathrm{ds}}^{\mathrm{e}}-I_{\mathrm{ds}}^{\mathrm{h}}$ is expressed through the well-known Landauer-Büttiker formalism [20]:

$$
I_{\mathrm{ds}}^{\mathrm{i}}=\frac{q}{\pi \hbar} \sum_{\alpha} \int_{E_{\alpha}^{\prime}(0)}^{E_{\alpha, \max }} \frac{T_{\mathrm{s}} T_{\mathrm{d}}}{T_{\mathrm{s}}+T_{\mathrm{d}}+T_{\mathrm{s}} T_{\mathrm{d}}}\left[f\left(\eta_{\alpha, \mathrm{s}}^{\mathrm{i}}\right)-f\left(\eta_{\alpha, \mathrm{d}}^{\mathrm{i}}\right)\right] \mathrm{d} E
$$

where $T_{\mathrm{s}}$ and $T_{\mathrm{d}}$ are transmittivities coefficients through the relevant Schottky barriers (equal to one if a barrier is not present), the $f$ functions are Fermi-Dirac distributions and electron density carrier can be determined by Fermi-Dirac distributions and Density of states, the coefficients $\eta_{\alpha, \mathrm{s}(\mathrm{d})}^{\mathrm{i}}$ are expressed as functions of the energy $E$ and the surface potential $\phi_{\mathrm{c}}$ :

$$
\eta_{\alpha, \mathrm{s}(\mathrm{d})}^{\mathrm{e}}=\frac{E-q \phi_{\mathrm{c}}-\mu_{\mathrm{s}(\mathrm{d})}}{k_{b} T}, \quad \eta_{\alpha, \mathrm{s}(\mathrm{d})}^{\mathrm{h}}=\frac{\mu_{\mathrm{s}(\mathrm{d})}-E+q \phi_{\mathrm{c}}}{k_{b} T}
$$


where $\mu_{\mathrm{s}}$ and $\mu_{\mathrm{d}}$ are the Fermi levels of source and drain, respectively. All the quantities in (14) and (15) are computed in the presence of the same relative deformation $d$.

In order to compute $I_{\mathrm{ds}}$ through (14), the surface potential $\phi_{\mathrm{c}}$ must be determined. If we limit ourselves to the case of ballistic FET, a constant potential can be assumed along the channel. The case of partially-ballistic FET can be solved in a modified way, not carrying out complications connected to the presence of a deformation, and for the sake of brevity it will not be explicitly treated here.

The surface potential $\phi_{\mathrm{c}}$ can then be determined by imposing a consistency relation between the mobile charges in the channel computed on a quantum-mechanical level $Q_{\text {micro }}\left(\phi_{\mathrm{c}}\right)$, and the $Q_{\text {macro }}\left(\phi_{\mathrm{c}}\right)$ through a macroscopic electrostatic model: $Q_{\text {micro }}\left(\phi_{\mathrm{c}}\right)=Q_{\text {macro }}\left(\phi_{\mathrm{c}}\right)$. The microelectronic model can be expressed as:

$$
Q_{\text {micro }}^{\mathrm{i}}=q \sum_{\alpha} \int_{E_{\alpha}^{\prime}(0)}^{E_{\alpha, \max }} \frac{T_{\mathrm{s}}\left(2-T_{\mathrm{d}}\right) f\left(\eta_{\alpha, \mathrm{s}}^{\mathrm{i}}\right)+T_{\mathrm{d}}\left(2-T_{\mathrm{s}}\right) f\left(\eta_{\alpha, \mathrm{d}}^{\mathrm{i}}\right)}{T_{\mathrm{s}}+T_{\mathrm{d}}+T_{\mathrm{s}} T_{\mathrm{d}}} D_{\alpha}^{\prime}(E) \mathrm{d} E
$$

with i $=\mathrm{e}, \mathrm{h}$, and $Q_{\text {micro }}=Q_{\text {micro }}^{\mathrm{h}}-Q_{\text {micro }}^{\mathrm{e}}$. The quantum-mechanical approach can be followed by computing all the quantities (effective mass, density of states, transmission coefficients) derived in this paper in the presence of a deformation. Its formulation takes into account Schottky barriers through the coefficients $T_{\mathrm{S}}$ and $T_{\mathrm{d}}$. It assumes a fully ballistic regime: the charges do not suffer from scattering by atoms in the channel, due to the reduced length of the channel. Longer channels in partially ballistic regime can be studied with a modified formulation; since the effects on geometrical deformation are modeled in the same way as described in the fully ballistic regime, we are limiting the presentation to the simpler case for the sake of brevity.

The macroscopic electrostatic model requires the knowledge of the capacitances $C_{\mathrm{g}}, C_{\mathrm{s}}, C_{\mathrm{c}}$ among the transistor contacts:

$$
Q_{\text {macro }}\left(\phi_{\mathrm{c}}\right)=\sum_{\mathrm{i}=\mathrm{g}, \mathrm{s}, \mathrm{d}} C_{\mathrm{i}}\left(V_{\mathrm{i}}-V_{\mathrm{FB}, \mathrm{i}}-\phi_{\mathrm{c}}\right)
$$

where $V_{i}$ are the voltage of gate, source and drain, respectively, and $V_{\mathrm{FB}, \mathrm{i}}$ are the relevant flatband voltages. Any deformation along the source-drain direction will cause a deformation along the vertical direction too, expressed through the Poisson coefficient $\nu_{\mathrm{SiO}_{2}}$ of the $\mathrm{SiO}_{2}$ dielectric, and a deformation along the nanoribbon width, expressed through the Poisson coefficient of the graphene $\nu$ [21]. Such modifications of the oxide thickness $t_{\mathrm{SiO}_{2}}^{\prime}$ and of the nanoribbon width $w^{\prime}$ will change the capacitance $C_{\mathrm{g}}\left(t_{\mathrm{SiO}_{2}}^{\prime}, w^{\prime}\right)=C_{\mathrm{g}}\left[t_{\mathrm{SiO}_{2}}\left(1-\nu_{\mathrm{SiO}_{2}} d\right), w(1-\nu d)\right]$, computed as suggested in [22] in closed-form through a semi-empirical formula, taking into account the fringing fields between gate and nanoribbon, dominant at this short scale.

The equality between the charges (16) and (17) leads to a nonlinear equation, which must be solved by means of the fixed-point method.

\section{NUMERICAL RESULTS}

The computation of the integrals in (14) finally allows a quantification of the effects of mechanical stresses on the performance of the complete transistor with an aGNR ballistic channel. In this section we show these effects in both the case of Schottky and Ohmic contacts at the interfaces drain-graphene and graphene-drain. Different ranges of relative deformation $d$ are studied in order to discuss their practical effects on the computed current. Small values of $d(d<0.1)$ are associated with applications of this class of devices to flexible electronics. As it will be confirmed in next results, current variations due to this range of deformation is not expected to prevent the functioning of the device for practical applications in electronic circuits.

In Figs. 6 we plot the variation of the channel potential $\phi_{\mathrm{c}}$ in different contact configurations and voltage excitation. The potential is numerically computed by enforcing the equation between (16) and (17). In Fig. 6(a) and (b) Ohmic contacts are considered, and $\phi_{\mathrm{c}}$ is computed when the gate 


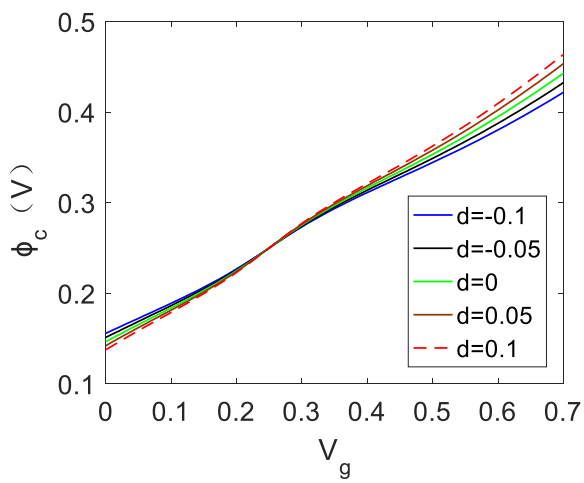

(a)

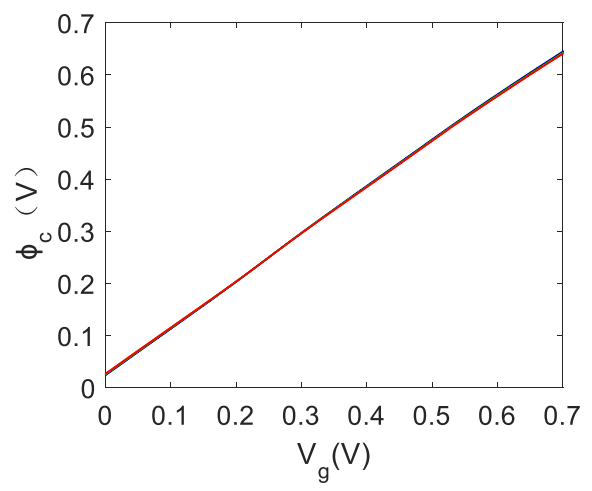

(c)

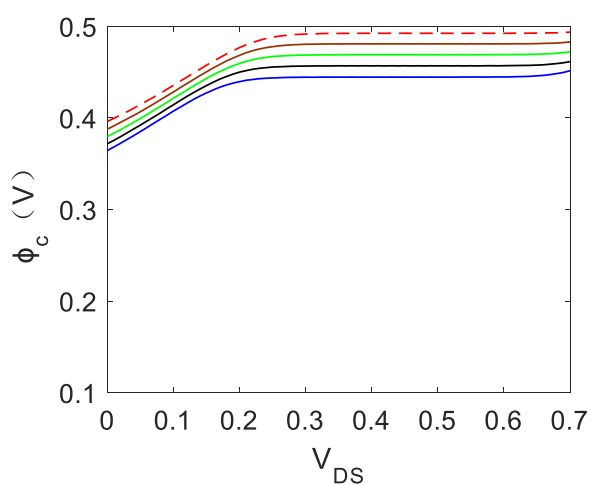

(b)

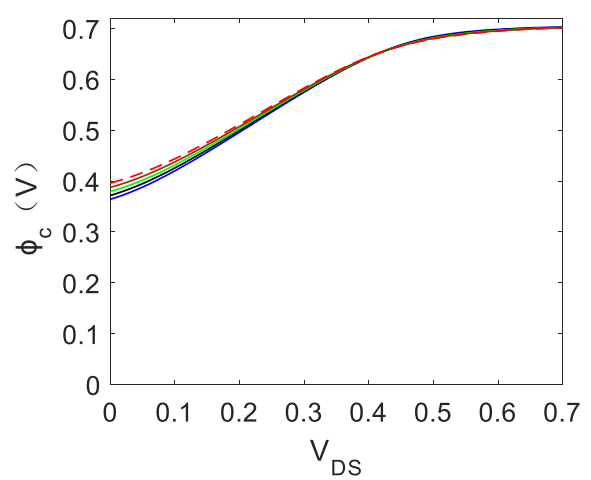

(d)

Figure 6. Channel potential $\phi_{\mathrm{c}}$. (a) $\phi_{\mathrm{c}}$ vs. $V_{\mathrm{g}}$, at $V_{\mathrm{ds}}=0.5 \mathrm{~V}$ with ohmic contacts. (b) $\phi_{\mathrm{c}} \mathrm{vs} . V_{\mathrm{ds}}$, at $V_{\mathrm{g}}=0.75$ $\mathrm{V}$ with ohmic contacts. (c) $\phi_{\mathrm{c}}$ vs. $V_{\mathrm{g}}$, at $V_{\mathrm{ds}}=0.5 \mathrm{~V}$ with $\mathrm{SB}$ contacts. (d) $\phi_{\mathrm{c}}$ vs. $V_{\mathrm{ds}}$, at $V_{\mathrm{g}}=0.75 \mathrm{~V}$ with SB contacts.

potential $V_{\mathrm{g}}$ and the drain-source potential $V_{\mathrm{ds}}$ is varied, respectively. The same results are shown in Figs. 6(c) and (d) in the presence of Schottky contacts. Different values of the relative deformation $d$ are shown in order to study the effect of $d$ on the potential values. The variation is very small for the Shottky contacts, while it is more evident in the case of Ohmic contacts.

The results for the potential lead to the computation of the charge stored inside the channel, computed by means of (17). In Fig. 7(a) and (b) we plot $Q$ in the absence of Schottky contacts, and in Fig. 7(c) and (d) the presence of Schottky contacts is considered. Consistently with the previous results, a smaller variation with $d$ is obtained for the Shottky with respect to the Ohmic contacts. The small but visible charge variation in the Shottky case is mainly due to the variation of the effective capacity $C_{g}$ due to the geometric deformation, since the potential $\phi_{\mathrm{c}}$ is more stable as shown in Figs. 6(c) and (d).

In Fig. 8(a) and (b) we plot the currents $I_{\mathrm{ds}}$ in the absence of Schottky contacts. In Fig. 8(a), $I_{\mathrm{ds}}$ is varied by keeping a constant potential $V_{\mathrm{ds}}=0.5 \mathrm{~V}$ and changing the gate potential $V_{\mathrm{g}}$. In Fig. 8(b), $I_{\mathrm{ds}}$ is varied by keeping a constant gate potential $V_{\mathrm{g}}=0.75 \mathrm{~V}$ and changing the drainsource potential $V_{\mathrm{ds}}$. The expected typical behaviors of currents controlled by the gate voltage and a definite threshold $V_{\mathrm{g}}$ voltage are visible in the figures, and can be evaluated in a simple way with this approach. In the absence of deformation, the method agrees with results given in [12].

In Fig. 8(c) and (d) we plot the currents $I_{\mathrm{ds}}$ in the presence of Schottky contacts. This means that, for the determination of the surface potential $\phi_{\mathrm{c}}$, the transmission coefficients have been computed in the integral (14) with the deformed quantities presented in Section 2. In Fig. 8(c), $I_{\mathrm{ds}}$ is varied by keeping a constant potential $V_{\mathrm{ds}}=0.5 \mathrm{~V}$ and changing the gate potential $V_{\mathrm{g}}$. In Fig. 8(d), $I_{\mathrm{ds}}$ 


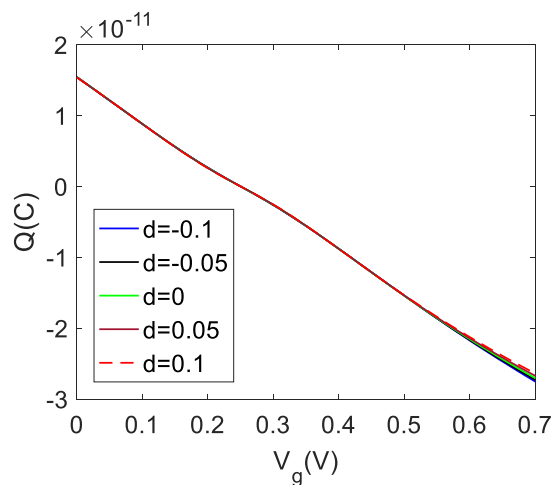

(a)

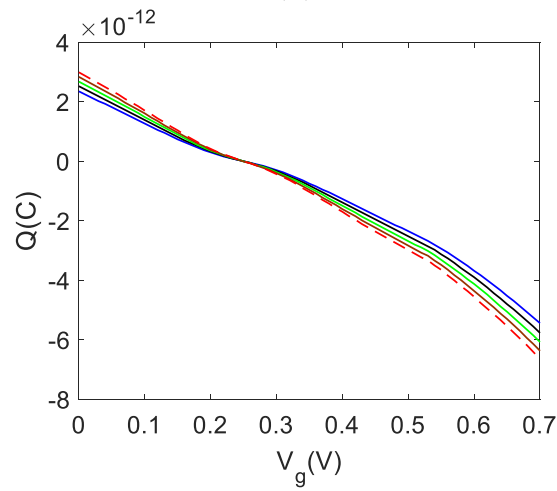

(c)

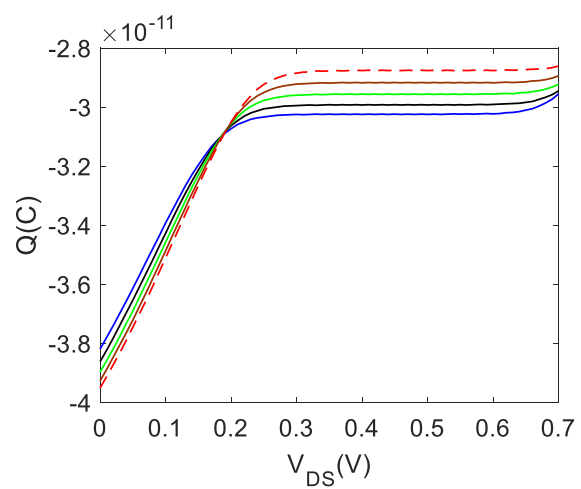

(b)

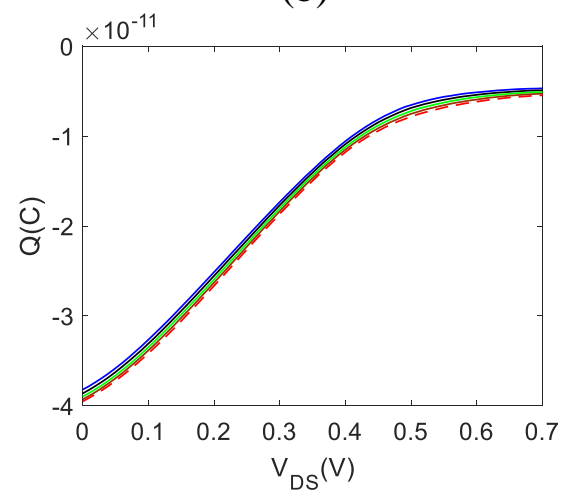

(d)

Figure 7. Macroscopic Charge $Q$. (a) $Q$ vs. $V_{\mathrm{g}}$, at $V_{\mathrm{ds}}=0.5 \mathrm{~V}$ with ohmic contacts. (b) $Q$ vs. $V_{\mathrm{ds}}$, at $V_{\mathrm{g}}=0.75$ V with ohmic contacts. (c) $Q$ vs. $V_{\mathrm{g}}$, at $V_{\mathrm{ds}}=0.5 \mathrm{~V}$ with $\mathrm{SB}$ contacts. (d) $Q$ vs. $V_{\mathrm{ds}}$, at $V_{\mathrm{g}}=0.75 \mathrm{~V}$ with SB contacts.

is varied by keeping a constant gate potential $V_{\mathrm{g}}=0.75 \mathrm{~V}$ and changing the drain-source potential $V_{\mathrm{ds}}$.

As expected, a lower level of currents is observed in these last two cases with respect to the previous ones, due to the presence of potential barriers limiting the probability of the charges to overcome the contact. Again, for small deformations current variations of about $10 \%$ is observed, while different results could be found for larger deformations. If compared with the values of $\phi_{\mathrm{c}}$ and $Q$ computed in Figs. 6 and 7, the variation of the currents appear more pronounced. This means that this variation with the deformation $d$ is mainly due to the variation of the transmittivity through the contacts (described by the coefficients $T_{\mathrm{d}}$ and $T_{\mathrm{s}}$ ), rather than a variation of the channel potential and charge.

An important remark concerns the computational cost of the method. The computation of $I_{\mathrm{ds}}$ requires the numerical solution of the equality $Q_{\text {micro }}\left(\phi_{\mathrm{c}}\right)=Q_{\text {macro }}\left(\phi_{\mathrm{c}}\right)$ to determine $\phi_{\mathrm{c}}$ and the computation of the integrals in (14). These tasks for a single value of $I_{\mathrm{ds}}$ in Fig. 8 requires about 0.7 seconds (on an Intel i7-4790 @ $3.60 \mathrm{GHz}$ ). Furthermore, no difficulties have been encountered in the solution of this equation when a deformation is introduced with respect to the standard case. The method is then proved to be a very effective approach to model geometrical deformations with both ballistic and nonballistic transport in the possible presence of Schottky barriers. An interesting feature of this approach is in fact that the geometrical parameters of the transistors appear explicitly in the formulation and can be tuned accordingly. 


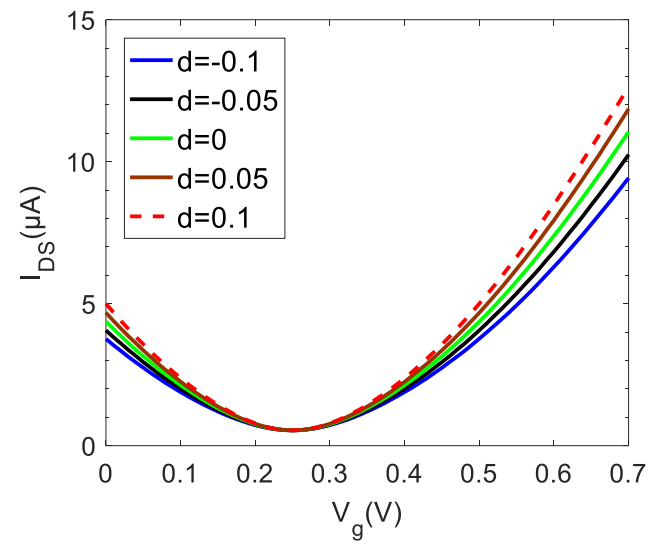

(a)

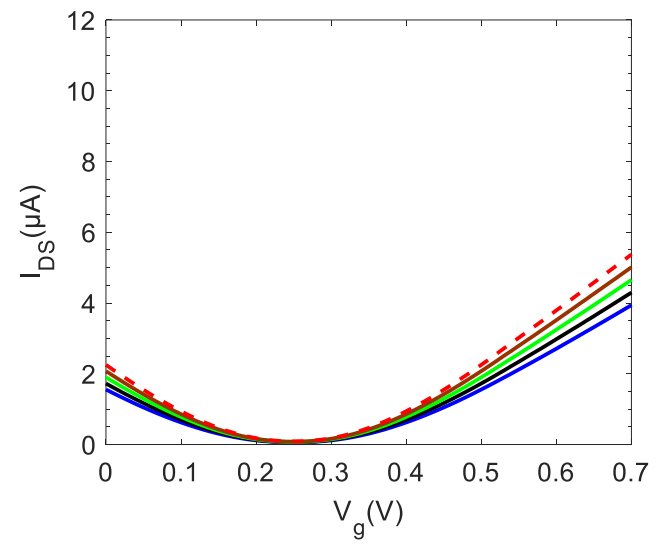

(c)

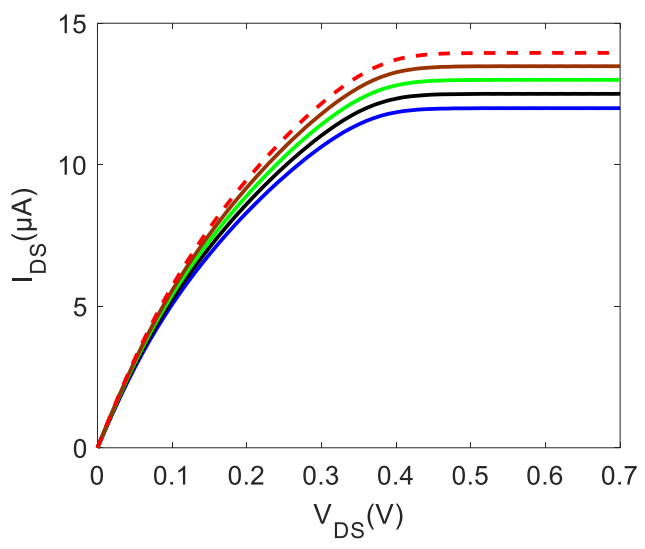

(b)

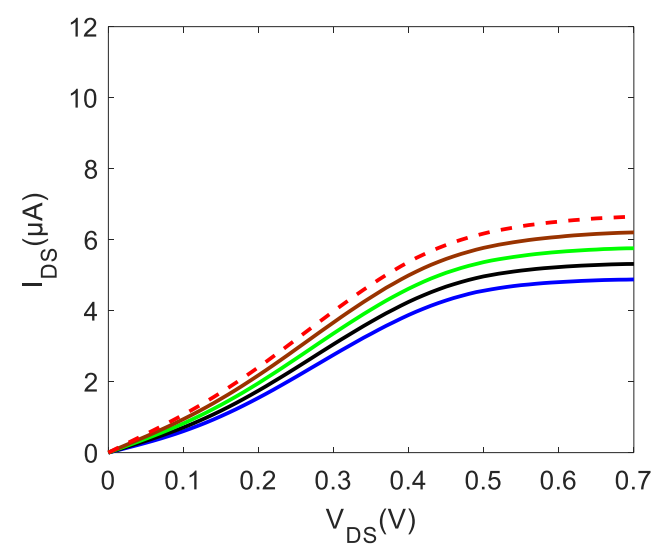

(d)

Figure 8 . Source-drain current $I_{\mathrm{ds}}$. (a) $I_{\mathrm{ds}}$ vs. $V_{\mathrm{g}}$, at $V_{\mathrm{ds}}=0.5 \mathrm{~V}$ with ohmic contacts. (b) $I_{\mathrm{ds}}$ vs. $V_{\mathrm{ds}}$, at $V_{\mathrm{g}}=0.75 \mathrm{~V}$ with ohmic contacts. (c) $I_{\mathrm{ds}}$ vs. $V_{\mathrm{g}}$, at $V_{\mathrm{ds}}=0.5 \mathrm{~V}$ with SB contacts. (d) $I_{\mathrm{ds}} \mathrm{vs} . V_{\mathrm{ds}}$, at $V_{\mathrm{g}}=0.75$ $\mathrm{V}$ with SB contacts.

\section{CONCLUSION}

The effect of mechanical deformations on graphene-based FETs has been investigated and quantified through a semi-analytical model of the energy bands of the deformed graphene nanoribbon. This kind of phenomena occurs in flexible electronics, where components can be submitted to different strains.

According to the result of the present study, the electronic characteristic of transistor is robust with respect to reasonable deformation cases in flexible electronics. For different applications, the drainsource currents could be modulated if required with a suitable tuning of higher deformation values $(d>0.1)$. However, in this last case mechanical stability of electrical connections could become an issue and it should be independently verified.

Future work will focus on the coupling of the present semi-analytic method with a more elaborate model replacing the tight-binding model used here, to take into account strain-related variations of energy gaps. Care will be given to propose an efficient method capable to handle parametric analyses of an entire FET, rather than severely computationally demanding method. Finally, practical realizations will be fabricated leading to an independent validations of the discussed results. 


\section{REFERENCES}

1. A. K. Geim and K. S. Novoselov, The rise of graphene, Nat. Mater., 6 (2007) 18391.

2. Wong and Akinwande, Carbon nanotube and Graphene Device Physics. Cambridge University Press, 2011.

3. D. Tien, J.Park, K. Kim, N. Lee and Y. Seo, Characterization of Graphene based FET Fabricated using a Shadow Mask, Scientific Reports, 6, 25050, 2016.

4. Z. H. Ni, T. Yu, Y. H. Lu, Y. Y. Wang, Y. P. Feng, and Z. X. Shen, Uniaxial strain on graphene: Ramen spectroscopy study and band-gap opening, ACS Nano 2, 2301-2305, 2008.

5. M. S. Sarker, M. M. Islam, M. R. Islam, an analytical approach to study energy band structure in strained graphene, Electrical Information and Communication Technology (EICT), 2013.

6. G. Gui, J. Li and J. Zhong, Band structure engineering of graphene by strain: First-principles calculations, Phys. Rev. $B, 78,075435,2008$.

7. G. Fiori and G. Iannaccone, Simulation of graphene nanoribbon field effect transistors, IEEE Electron Devices Lett., vol. 28, no. 8, pp. 760762, Aug. 2007.

8. L. Sun, Q. Li, H. Ren, Q. W. Shi, J. Yang, and J. G. Hou, Strain effect on energy gaps of armchair graphene nanoribbons, e-print arXiv: condmat/0703795.

9. Y. Li, X. Jiang, Z. Liu, Z. Liu, Strain Effects in Graphene and Graphene Nanoribbons: The Underlying Mechanism, Nano Research, vol. 3, no. 8, pp. 545-556, 2010.

10. K. Alam, Uniaxial Strain Effects on the Performance of a Ballistic Top Gate Graphene Nanoribbon on Insulator Transistor, IEEE Trans. Nanotechnol., vol. 8, pp. 528534, 2009.

11. M. R. Moslemi, M. H. Sheikhi, K. Saghafi, M. K. Moravvej-Farshi, Electronic properties of a dual-gated GNR-FET under uniaxial tensile strain, Microelectronics Reliability, vol. 52, pp. 25792584, 2012.

12. P. Michetti and G. Iannaccone, Analytical model of one-dimensional carbon-based Schottky-barrier transistors, IEEE Trans. Electron Devices, vol. 57, no. 7, pp. 16161625, Jul. 2010.

13. J.M. Ziman, Principles of the theory of solids, Cambridge University Press, 1965. Press, Cambridge, 1995.

14. N.W. Ashcroft, N.D. Mermin, Solid State Physics, Holt Saunders International Edition, 1976.

15. D. Pettifor, Bonding and structure of molecules and solids, Oxford Science Publication, 1995.

16. P. Kireev, Semiconductor Physics, Mir Publishers, 1976.

17. R. Roldn, M. P. Lopez-Sancho, and F. Guinea, Effect of electron-electron interaction on the Fermi surface topology of doped graphene, Phys. Rev. B, 6, 25050, 2016.

18. Y. Li, X. Jiang, Z. Liu, and Z. Liu, Strain Effects in Graphene and Graphene Nanoribbons: The Underlying Mechanism, Nano Research., 3(8): 545556, August 2010.

19. Y. W. Son, M. Cohen, and S. Louie, Energy gaps in graphene nanoribbons, Phis. Rev. Lett., vol. 97, no. 21, p. 216 803, Nov. 2006.

20. S. Datta, Electronic Transport in Electronic Systems. Cambridge University Press, Cambridge, 1995.

21. R. Faccio, P. A. Denis, H. Pardo, C. Goyenola, A. W. Mombru, Mechanical properties of graphene nanoribbons, $J$. Physics: Cond. Matt., 21(8), 285304, 2009.

22. A. Behnam, A. S. Lyons, M.-H. Bae, E. K. Chow, S. Islam, C. M. Neumann, and E. Pop, Transport in nanoribbon interconnects obtained from graphene grown by chemical vapor deposition, Nano Lett. 2012, 12, 4424. 